\title{
BEBERAPA KONSEP YANG BERKAITAN PADA RUANG FUZZY BERNORMA DAN RUANG FUZZY BERNORMA-n
}

\author{
Mashadi \\ Jurusan Matematika FMIPA Univeristas Riau \\ mash-mat@unri.ac.id
}

\begin{abstract}
In this paper we will discuss some of the approaches of fuzzy normed spaces and development on fuzzy n-normed space. The approach discussed is based on the concept of t-norm and-t conorm and directly as well as defining the usual definition normed space. But it is discussed in more detail in this paper is the definition that refers to a generalized regular normed spaces on fuzzy 2-normed space and space and fuzzy n-normed space.
\end{abstract}

Key words :Fuzzy Normed Space, Fuzzy 2-normed Space, Fuzzy n-normed Space, Fuzzy Point

ABSTRAK. Pada makalah ini akan dibahas beberapa pendekatan dari ruang fuzzy bernorma dan pengembangannya pada ruang fuzzy bernorma-n. Pendekatan yang dibahas adalah berdasarkan konsep norma-t dan conorma-t serta pendefinisian langsung seperti pendefinisian ruang bernorma biasa. Akan tetapi yang dibahas lebih detil dalam tulisan ini adalah pendefinisian yang mengacu pada ruang bernormaa biasa yang diperumum pada ruang fuzzy bernorma-2 dan ruang fuzzy bernorma- $n$.

Kata kunci : Ruang Fuzzy Bernorma, Ruang Fuzzy Bernorma-2, Ruang Fuzzy Bernorma-n. Titik Fuzzy.

\section{PENDAhUluan}

Teori himpunan fuzzy pertama sekali diperkenalkan oleh L. Zadeh tahun 1965. Sampai saat ini berkembang banyak sekali penggunaan dari himpunan dan bilangan fuzzy tersebut, Dalam ilmu matematika perkembangannya tidak hanya dalam bidang Analisis saja, akan tetapi dalam semua bidang kajian yang ada dalam matematika, termasuk dalam bidang Numerik, Operasi Riset, Statistik, Aljabar dan aljabar linear (Alotaibi, 2010; Elegan, dkk, 2010; Karakus, dkk, 2008, Mashadi, 2010).

Dalam bidang matematika analisis, konsep fuzzy juga sangat berkembang, sampai pada masalah fuzzy ruang metric-n dan ruang fuzzy bernorma- $n$. Konsep ruang fuzzy bernorma dan ruang fuzzy bernorma-2 sampai ke ruang fuzzy bernorma$n$, sebenarnya didekati dari 2 sisi, yang pertama yaitu ruang fuzzy bernorma yang 
didekati secara Intuitionistic yaitu dengan menggunakan konsep konntinu t-norm dan kontinu t-conorm (Alotaibi, 2010; Golet, 2009; Mursaleen dan Danish Lohani, 2008; Samanta dan Mohinta, 2011; Surender, 2011; Vaezpour dan Karini, 2008). Dipihak lain ada juga penulis yang memperkenalkan konsep ruang fuzzy bernorma menggunakan pendekatan fuzzy point dari himpunan fuzzy.

\section{RUANG FUZZY BERNORMA}

Seperti yang disebutkan di atas, salah satu pendekatan ruang fuzzy bernorma adalah secara intuitionistic, yaitu konsep ruang fuzzy bernorma yang didekati berdasarkan konsep kontinu $t$-norm dan kontinu $t$-conorm (Alotaibi, 2010; Golet, 2009; Mursaleen dan Danish Lohani, 2008; Samanta dan Mohinta, 2011).

Definisi 2.1. Misalkan $\mathrm{X}$ himpunan tak kosong, Himpunan Fuzzy $\tilde{A}$ di $\mathrm{X}$ adalah suatu himpunan yang fungi keanggotaannya, $\mu_{\tilde{A}}: \mathrm{X} \rightarrow[0,1]$.

Sehingga $\tilde{A}$ dapat ditulis dalam bentuk $\tilde{A}=\left\{\left(x, \mu_{\tilde{A}}(x)\right) \mid x \in X, 0 \leq \mu_{\tilde{A}}(x) \leq 1\right\}$

Definisi 2.2. suatu operasi biner $*[0,1] \times[0,1] \rightarrow[0,1]$ dikatakan kontinu $t$-norm jika memenuhi kondisi berikut :

i. * assiosiati dan komutatif

ii. * kontinu

iii. $a * 1=a$ untuk semua $a \in[0,1]$

iv. $a * b \leq c * d$ bila $a \leq c$ dan $b \leq d$ untuk setiap $a, b, c, d[0,1]$.

Maka jelas jika didefinisikan $a * b=a b$ atau $a * b=\{a, b\}$, maka $*$ merupakan kontinu $t$-norm. 
Definisi 2.3. suatu operasi biner $\diamond:[0,1] \times[0,1] \rightarrow[0,1]$ dikatakan kontinu $t-$ conorm jika memenuhi kondisi berikut :

i. $\diamond$ assosiatif dan komutatif

ii. $\diamond$ kontinu

iii. a $\searrow 0=0$ untuk semua $a \in[0,1]$

iv. $a \diamond b \leq c \diamond d$ bila $a \leq c$ dan $b \leq d$ untuk setiap $a, b, c, d[0,1]$.

Definisi 2.4. Lima tupelo $(X, \mu, \nu, *, \diamond)$ dikatakan Instutionistic Ruang fuzzy bernorma (disingkat IFNS), jika $\mathrm{X}$ adalah ruang vektor, $*$ kontinu $t$-norm, $\diamond$ kontinu $t$-conorm dan $\mu, \nu$ adalah himpunan fuzzy pada $X \times[0, \infty]$ yang memenuhi kondisi, untuk setiap $x, y \in \mathrm{X}$ dan $s, t>0$

(a). $\mu(x, t)+v(x, t) \leq 1$

(b). $\mu(x, t)>0$

(c). $\mu(x, t)=1$ jika dan hanya jika $x=0$

(d). $\mu(\propto x, t)=\mu\left(x, \frac{t}{\alpha}\right)$ untuk setiap $\alpha \neq 0$.

(e). $\mu(x, t) * \mu(y, s) \leq \mu(x+y, t+s)$

(f). $\mu(x,):.[0, \infty) \rightarrow[0,1]$ kontinu

(g). $\lim _{t \rightarrow \infty} \mu(x, t)=1$ dan $\lim _{t \rightarrow 0} \mu(x, t)=0$

(h). $v(x, t)<1$,

(i). $v(x, t)=0$ jika dan hanya jika $x=0$

(j). $v(\propto x, t)=v\left(x, \frac{t}{\alpha}\right)$ untuk setiap $\alpha \neq 0$ 
(k). $v(x, t) \diamond(y, s) \leq v(x+y, t+s)$

(l). $v(x,):.[0, \infty) \rightarrow[0,1]$ kontinu

(m). $\lim _{t \rightarrow \infty} v(x, t)=0$ dan $\lim _{t \rightarrow 0} v(x, t)=1$

Dalam hal ini $\mu, \nu$ dikatakan Intuitionistic Fuzzy Norma.

Beberapa penulis (Saadi dan Vaezpour, 2005; Sharma, 2002; Surender, 2011; Vaezpour dan Karini, 2011) justru mendefinisikan ruang fuzzy bernorma hanya dengan memberikan syarat yang lebih sederhana yaitu sebagai berikut.

Definisi 2.5. Pasangan 3-tupel $(X, V, *$, ) dikatakan Ruang fuzzy bernorma, jika $X$ adalah ruang vektor, $*$ kontinu $t$-norm dan $V$ adalah himpunan fuzzy pada $\mathrm{X} \times[0, \infty]$ yang memenuhi kondisi, untuk setiap $x, y \in \mathrm{V}$ dan $s, t>0$ berlaku

(i). $V(x, t)>0$

(ii). $V(x, t)=1$ jika dan hanya jika $x=0$

(iii). $V(\propto x, t)=V\left(x, \frac{t}{\alpha}\right)$ untuk setiap $\alpha \neq 0$.

(iv). $V(x, t) * V(y, s) \leq \mathrm{V}(x+y, t+s)$

(v). $V(\mathrm{x},):.[0, \infty) \rightarrow[0,1]$ kontinu

(vi). $\lim _{t \rightarrow \infty} \mu(x, t)=1$

Akan tetapi ada juga penulis lain yang mengganti syarat (i). (iv) dan (v) sebagai berikut :

(i'). $V(x, t)=0$ untuk setiap $t \leq 0$. (sebenarnya syarat ini ekivalen dengan syarat (i)).

(iv'). $V(\mathrm{x}+\mathrm{y}, \mathrm{s}+\mathrm{t}) \geq \min \{V(\mathrm{x}, \mathrm{s}), V(\mathrm{y}, \mathrm{t})\}$.

Syarat (iv') ini sebenarnya juga ekivalen dengan syarat (iv), karena salah satu contoh dari kontinu $t$-norm adalah $a * b=\{a, b\}$.

(v'). $V(\mathrm{x},$.$) merupakan fungsi tak turun di \mathrm{R}$, yang sebenarnya juga ekivelan dengan syarat $(\mathrm{v})$, 
Bila $(X,\|\|$.$) ruang bernorma dan didefinisikan a * b=a b$ atau $a * b=\min$ $\{a, b\}$, kemudian definisikan

$$
V(x, t)=\left\{\begin{array}{cl}
\frac{k t^{n}}{k t^{n}+m\|x\|}, & \text { bila } \mathrm{t}>0 \text { dan } k, m, n \in \mathrm{R}^{+} \\
0 & \text { bila } t \leq 0
\end{array}\right.
$$

Maka $(\mathrm{X}, V, *)$ adalah ruang bernormaa fuzzy. Khususnya jika $k=n=m=1$ akan diperoleh

$$
V(x, t)= \begin{cases}\frac{t}{t+\|x\|}, & \text { bila } t>0 \\ 0, & \text { bila } t \leq 0\end{cases}
$$

Yang merupakan norma fuzzy standart yang di induksi dari norma \|. \|.

Bila $(X,\|\|$.$) ruang bernormaa dan didefinisikan a * b=a b$ dengan

$$
V(x, t)=\left(\exp \frac{\|x\|}{t}\right)^{-1}
$$

Untuk semua $x \in \mathrm{X}$ dan $\mathrm{t} \in(0, \infty)$. Maka $(\mathrm{X}, V, *)$ adalah ruang fuzzy bernorma.

Definisi 2.6. Misalkan $(\mathrm{X}, V, *)$ adalah ruang fuzzy bernorma, misalkan $\left\{x_{n}\right\}$ barisan di $V$, maka barisan $\left\{x_{n}\right\}$ dikatakan konvergen, jika terdapat $x \in \mathrm{V}$ sehingga $\lim _{n \rightarrow \infty} V\left(x_{n}-x, t\right)=1$, untuk setiap $t>0$.

Definisi 2.7. Misalkan $(X, V, *)$ adalah ruang fuzzy bernorma, misalkan $\left\{x_{n}\right\}$ barisan di $V$, maka barisan $\left\{x_{n}\right\}$ dikatakan barisan Cauchy, jika $\lim _{n \rightarrow \infty} V\left(x_{n+p}-x_{n}, t\right)=$ 1 , untuk setiap $t>0$ dan $p=1,2,3, \ldots$

Dari definisi di atas juga akan berlaku bahwa dalam ruang fuzzy bernorma, setiap barisan yang konvergen juga akan merupakan barisan Cauchy.

Definisi 2.8. Misalkan $\mathrm{A} \subseteq \mathrm{X}$ dengan $(\mathrm{X}, V, *)$ adalah ruang fuzzy bernorma, $\mathrm{A}$ dikatakan terbatas jika dan hanya jika terdapat $\mathrm{t}>0$ dan $0<r<1$ sehingga $V(x, t)>1$ $-\mathrm{r}$ untuk semua $x \in \mathrm{A}$. 
Pendekatan lain untuk ruang fuzzy bernorma adalah sebagai berikut, dimulai dengan mendefinisikan titik fuzzy $\mathrm{P}_{x}$ pada $\mathrm{X}$ yaitu sebagai berikut (Iqbal dan Hemen, 2010; Karakus, dkk, 2008).

Definisi 2.9. Suatu titik fuzzy $P_{x}$ di $X$ adalah himpunan fuzzy dengan fungsi keanggotaan

$$
\mu_{P x}(y)=\left\{\begin{array}{c}
\propto, \quad \text { jika } y=x \\
0, \text { untuk lainnya }
\end{array}\right.
$$

Untuk semua $y \in \mathrm{X}$ dengan $0<\alpha<1$. Kita notasikan titik fuzzy dengan $x_{\alpha}$ atau $(x, \alpha)$.

Misalkan X ruang vektor atas lapangan $\mathrm{K}$, dan misalkan $\tilde{A}$ himpunan fuzzy di $\mathrm{X}$, maka $\tilde{A}$ dikatakan sub ruang fuzzy di $\mathrm{X}$ jika untuk semua $x, y \in \mathrm{X}$ dan $\lambda \in \mathrm{K}$ berlaku

i. $\quad \mu_{\tilde{A}}(x+y) \geq \min \left\{\mu_{\tilde{A}}(x), \mu_{\tilde{A}}(y)\right\}$

ii. $\quad \mu_{\tilde{A}}(\lambda x) \geq \mu_{\tilde{A}}(x)$.

Berdasarkan konsep di atas didefinisikanlah ruang fuzzy bernorma yaitu sebagai berikut :

Definisi 2.10. Misalkan $X$ ruang vector atas lapangan $K$, dan misalkan $\|.\|_{f}: X \rightarrow[0$, $\infty)$, fungsi yang mengaitkan setiap titik $x_{\alpha}$ di $X, \alpha \in(0,1]$ bilangan real tak negatip \|. $\|_{f}$ sehingga :

(FN1). $\left\|x_{\alpha}\right\|_{f}=0$ jika dan hanya jika $x=0$

(FN2). $\left\|\lambda x_{\alpha}\right\|_{f}=|\lambda|\left\|x_{\alpha}\right\|$, untuk semua $\lambda \in \mathrm{K}$.

(FN3). $\left\|x_{\alpha}+y_{\beta}\right\|_{f} \leq\left\|x_{\alpha}+y_{\beta}\right\|_{f}$

(FN4). Jika $\left\|x_{\alpha}\right\|_{f}<r$, dengan $r>0$ maka terdapat $0<\sigma \leq \alpha<1$ sehingga $\left\|x_{\alpha}\right\|_{f}<$ $r$.

Maka $\|.\|_{f}$ disebut fuzzy norma dan $\left(X . \widetilde{A},\|.\|_{f}\right)$ disebut ruang fuzzy bernorma. 
Berdasarkan Definisi 2.10 di atas, maka didapat hubungan antara rnorma biasa dengan fuzzy norm yaitu sebagai berikut.

Proposisi 2.11.Misalkan (X.\|.\|) ruang bernorma biasa, definisikan $\left\|x_{\alpha}\right\|_{f}=$ $\frac{1}{\alpha}\|x\|$ untuk semua $x_{\alpha} \in X$ dengan $\alpha \in(0,1]$, maka $\left(X . \widetilde{A},\|.\|_{f}\right)$ merupakan ruang fuzzy bernorma.

Bukti : Misalkan $x_{\alpha}$ dan $y_{\beta} \in \widetilde{A}$ dengan $\alpha, \beta \in(0,1]$ dan $\lambda \in \mathrm{K}$. maka :

(FN1). $\left\|x_{\alpha}\right\|_{f}=0 \Leftrightarrow \frac{1}{\alpha}\|x\|=0 \Leftrightarrow\|x\|=0 \Leftrightarrow \mathrm{x}=0$.

(FN2). $\left\|\lambda x_{\alpha}\right\|_{f}=\frac{1}{\alpha}\|\lambda x\|=\frac{|\lambda|}{\alpha}\|x\|=|\lambda| .\left\|x_{\alpha}\right\|_{f}$

(FN3). $\left\|x_{\alpha}+y_{\beta}\right\|_{f}=\left\|(x+y)_{\gamma}\right\|_{f}$, dengan $\gamma=\operatorname{maks}\{\alpha, \beta\}$

$$
=\frac{1}{\gamma}\|x+y\| \leq \frac{1}{\gamma}\|x\|+\frac{1}{\gamma}\|y\| \leq \frac{1}{\alpha}\|x\|+\frac{1}{\beta}\|y\|=\left\|x_{\alpha}\right\|_{f}+
$$

$\left\|y_{\beta}\right\|_{f}$

(FN4). Jika $\left\|x_{\alpha}\right\|_{f}<r$, dengan $r>0$, maka untuk $\rho \in(0,1]$ dengan $\alpha \leq \rho$, maka berlaku :

$$
\frac{\|x\|}{\rho} \leq \frac{\|x\|}{\alpha}<r \text { yang bermakna }\left\|x_{\alpha}\right\|_{f}<r .
$$

Sebaliknya bila $\left\|x_{\alpha}\right\|_{f}$ adalah suatu Ruang fuzzy bernorma, definisikan $\|x\|=\left\|x_{1}\right\|_{f}=\|(x, 1)\|_{f}$ merupakan ruang bernorma. Jadi bila kita punya ruang bernorma, maka senantiasa bisa kita bentuk ruang fuzzy bernorma dan begitu juga sebaliknya, dengan kata lain ruang bernorma adalah ekivalen dengan ruang fuzzy bernorma. Sehingga semua sifat yang berlaku pada ruang bernorma juga akan berlaku pada ruang fuzzy bernorma.

Selanjutnya (Kider, 2011a dan 2011b), mendefinisikan himpunan fuzzy buka, untuk himpunan fuzzy $\widetilde{A}$ di X, himpunan fuzzy tutup dan fuzzy kontinu di $\widetilde{A}$ didefinisikan sebagai berikut. 
Definisi 2.12. Misalkan $\left(X . \widetilde{A},\|.\|_{f}\right)$ ruang fuzzy bernorma. Diberikan $x_{\alpha} \in \widetilde{A}$ dan bilangan real $r>0$. Maka $\left.\widetilde{B(} x_{\alpha}, r\right)=\left\{y_{\beta} \in \widetilde{A}:\left\|x_{\alpha}-y_{\beta}\right\|_{f}<r\right\}$ dikatakan bola buka dengan jari-jari $r$.

Berdasarkan Definisi 2.12 di atas, maka dapatlah didefinisikan himpunan buka dan himpunan tutup pada suatu ruang fuzzy bernorma. Misalkan $\widetilde{A}$ himpunan fuzzy pada ruang fuzzy bernorma $\left(X . \widetilde{A},\|.\|_{f}\right)$ dikatakan buka jika setiap $x_{\alpha} \in \widetilde{A}$ terdapat $\widetilde{B(}\left(x_{\alpha}, r\right) \subseteq \widetilde{A}$ dan himpunan fuzzy $\widetilde{B}$ dikatakan tutup jika komplementnya buka.

Definisi 2.13. Misalkan $\left(X . \widetilde{A},\|.\|_{f_{1}}\right)$ dan $\left(Y . \widetilde{B},\|.\|_{f_{2}}\right)$ masing-masing ruang fuzzy bernorma. Pemetaan $\mathrm{T}: \mathrm{X} \rightarrow \mathrm{Y}$ dikatakan fuzzy kontinu di $x_{\alpha} \in \widetilde{A}$ jika untuk setiap $\varepsilon>0$ terdapat $\delta>0$ sehingga $\left\|T(x)_{\alpha}-T(y)_{\beta}\right\|_{f_{2}}<\varepsilon$ untuk semua $y_{\beta} \in \tilde{B}$ dengan $\left\|x_{\propto}-y_{\beta}\right\|_{f_{1}}<\delta$

$\mathrm{T}$ dikatakan fuzzy kontinu jika $\mathrm{T}$ fuzzy kontinu disetiap titik $x_{\alpha} \in \widetilde{A}$. Selanjutnya dengan menggunakan Definisi 2.13 di atas akan dapat ditunjukkan pemetaan $\mathrm{T}$ dari ruang fuzzy bernorma $\left(X . \widetilde{A},\|.\|_{f_{1}}\right)$ into ruang fuzzy bernorma $\left(Y . \widetilde{B},\|.\|_{f_{2}}\right)$ adalah fuzzy kontinu jika dan hanya jika setiap inverse image dari sebarang himpunan fuzzy buka di $\tilde{B}$ adalah buka di X.

Selanjutnya didefinisikan barisan konvergen, himpunan terbatas pada ruang fuzzy bernorma

Definisi 2.14. Barisan fuzzy $\left\{\left(x_{n}, \propto_{n}\right)\right\}$ pada ruang fuzzy bernorma $\left(X . \widetilde{A},\|.\|_{f}\right)$ dikatakan konvergen $x_{\propto} \in \widetilde{A}$, jika $\lim _{n \rightarrow \infty}\left\|\left(x_{n}, \propto_{n}\right)-x_{\propto}\right\|=0$, dan $x_{\propto}$ dikatakan limit dari $\left\{\left(x_{n}, \propto_{n}\right)\right\}$ dan ditulis $\lim _{n \rightarrow \infty}\left\{\left(x_{n}, \propto_{n}\right)\right\}=x_{\propto}$. 
Definisi 2.15. Barisan fuzzy $\left\{\left(x_{n}, \propto_{n}\right)\right\}$ pada ruang fuzzy bernorma $\left(X . \widetilde{A},\|.\|_{f}\right)$ dikatakan barisan Cauchy jika untuk setiap $\varepsilon>0$ terdapat $\mathrm{N}_{0}>0$ sehingga $\left\|\left(x_{n}, \propto_{n}\right)-\left(x_{m}, \propto_{m}\right)\right\|_{f}<\varepsilon$ untuk setiap $m, n>\mathrm{N}_{0}$.

Berdasarkan definisi-definisi di atas, maka teorema-teorema yang ada dalam barisan bilangan real akan berlaku untuk barisa pada ruang fuzzy bernorma (Kider, 2011a dan 2011b), misalnya setiap barisan convergen pada ruang linear bernorm fuzzy adalah barisan Cauchy. Begitu juga jika Pemetaan T $: X \rightarrow$ Y pada ruang fuzzy bernorma $\left(X . \widetilde{A},\|.\|_{f_{1}}\right)$ dan $\left(Y . \widetilde{B},\|.\|_{f_{2}}\right)$ adalah fuzzy kontinu di $x_{\alpha} \in \widetilde{A}$ jika dan hanya jika $\left\{\left(x_{n}, \propto_{n}\right)\right\} \rightarrow x_{\alpha}$ menyebabkan $\left\{T\left(x_{n}\right), \propto_{n}\right\} \rightarrow T(x)_{\alpha}$. Banyak lagi konsep lain yang juga akan berlaku, misalnya keterbatasan, kelengkapan.

\section{RUANG FUZZY BERNORMA-2.}

Sama seperti ruang fuzzy bernorma, banyak pendekatan yang dilakukan untuk pendefinisian ruang fuzzy bernorma-2, akan tetapi dalam tulisan ini konsep ruang fuzzy bernorma-2 hanya akan diperumum dari Definisi 2.5 dan 2.10.

Definisi 3.1. Pasangan 3-tupel $(X, V, *$, ) dikatakan ruang fuzzy bernorma-2 jika $X$ adalah ruang vector, $*$ adalah kontinu $t$-norm dan $\mathrm{V}$ himpunan fuzzy di $\mathrm{X} \times \mathrm{X} \times \mathrm{R}$ dan untuk setiap $\mathrm{x}, \mathrm{y} \in \mathrm{X}$ dan $\mathrm{s}, \mathrm{t}>0$ memenuhi.

(FN-3.1). $V(\mathrm{x}, \mathrm{y} ; \mathrm{t})=0$ untuk semua $t \in \mathrm{R}$ dan $t \leq 0$.

(FN-3.2). $V(x, y ; t)=1$, jika dan hanya jikax dan y bergangung linear, dengan $t \in \mathrm{R}, t$ $>0$.

(FN-3.3). $V(x, y ; t)=V(y, x ; t)$ untuk semua $x, y \in \mathrm{X}$..

(FN-3.4). $V(\alpha x, y ; t)=V\left(x, y ; \frac{t}{\alpha}\right)$ untuk setiap $\alpha \neq 0$.

$(\mathrm{FN}-3.5) . V(x, z ; s) * V(y, z ; t) \leq V(x+y, z ; s+t)$

$(\mathrm{FN}-3.6) . V(x, y ;):.(0, \infty) \rightarrow[0,1]$ fungsi takturun untuk $t \in \mathrm{R}$. 
(FN-3.7). $\lim _{t \rightarrow \infty} V(x, y ; t)=1$,

Maka $V$ dikatakan dikatakan norm-2 fuzzy pada $X$ dan pasangan $(X, V, *)$ dikatakan ruang fuzzy bernorma-2.

Mengacu pada contoh ruang fuzzy bernorma, maka Bila $(X,\|\bullet, \bullet\|)$ ruang bernorma-2, definisikan $a * b=a b$ atau $a * b=\min \{\mathrm{a}, \mathrm{b}\}$ dan

$$
V(x, y ; t)=\left\{\begin{array}{cc}
\frac{k t^{n}}{k t^{n}+m\|x, y\|} & \text { bila } k, m, n, t>0, \text { dan } x, y \in X \\
0, & \text { bila } t \leq 0, \text { dan } x, y \in R
\end{array}\right.
$$

Maka $(X, V, *)$ merupakan ruag fuzzy bernorm-2. Khusus jika $k=n=m=1$, maka diperoleh bentuk

$$
V(x, y ; t)=\left\{\begin{aligned}
\frac{t}{t+\|x, y\|} & \text { bila } t>0, \text { dan } x, y \in X \\
0, & \text { bila } t \leq 0, \text { dan } x, y \in
\end{aligned}\right.
$$

Yang disebut dengan norma-2 fuzzy standart dengan norma $\|\bullet, \bullet\|$.

Berikut ini diberikan contoh ruang fuzzy bernorma-2 yang tidak standar lainya, misalkan Bila $(X,\|\bullet, \bullet\|)$ ruang bernorma-2, definisikan $a * b=a b$ atau $a * b=$ $\min \{a, b\}$ dan definisikan :

$$
V(x, y ; t)= \begin{cases}\frac{t^{2}-\|x, y\|^{2}}{t^{2}+\|x, y\|^{2}} & \text { bila } t>\|x, y\| \\ 0, & \text { bila } t<\|x, y\|\end{cases}
$$

(FN-3.1). jika $t \leq\|x, y\|$ dan jelas dari definisi $V(x, y ; t)=0$, untuk setiap $x, y \in \mathrm{X}$.

(FN-3.2). untuk $t>0$ dengan $t>\|x, y\|$, misalkan $V(x, y ; t)=1$.

$$
\begin{aligned}
& \Leftrightarrow \frac{t^{2}-\|x, y\|^{2}}{t^{2}+\|x, y\|^{2}}=1 \\
& \Leftrightarrow t^{2}-\|x, y\|^{2}=t^{2}+\|x, y\|^{2} \\
& \Leftrightarrow\|x, y\|=0 \\
& \Leftrightarrow x \text { dan } y \text { bergantung linear. }
\end{aligned}
$$

(FN-3.3). Jelas berlaku dari definisi $\|x, y\|=\|y, x\|$

(FN-3.4). Untuk $\alpha \neq 0$ dan jika $\mathrm{t}>\|\propto x, y\|$, maka 


$$
V(\propto \mathrm{x}, \mathrm{y} ; \mathrm{t})=\frac{t^{2}-\|\propto x, y\|^{2}}{t^{2}+\|\propto x, y\|^{2}}=\frac{t^{2}-|\propto|^{2}\|x, y\|^{2}}{t^{2}+|\propto|^{2}\|x, y\|^{2}}=\frac{\left(\frac{t}{|\alpha|}\right)^{2}-\|x, y\|^{2}}{\left(\frac{t}{|\alpha|}\right)^{2}+\|x, y\|^{2}}=V\left(x, y ; \frac{t}{|\alpha|}\right)
$$

Untuk $\alpha \neq 0$ dan jika $\mathrm{t} \leq\|\propto x, y\|$, maka $\frac{t}{|\propto|} \leq\|x, y\|$, jadi

$$
V(\propto x, y ; t)=0=V\left(x, y ; \frac{t}{|\propto|}\right)
$$

(FN-3.5). Misalkan $s \leq\|x, z\|$ atau $t \leq\|y, z\|$, maka jelas ketaksamaan dipenuhi. Selanjutnya misalkan $s>\|x, z\|$ dan $t>\|y, z\|$, dan tanpa mengurangi keumuman misalkan $V(y, z ; t) \geq V(x, y ; t)$ maka diperoleh

$$
\begin{aligned}
& \frac{t^{2}-\|y, z\|^{2}}{t^{2}+\|y, z\|^{2}} \geq \frac{s^{2}-\|x, z\|^{2}}{s^{2}+\|x, z\|^{2}} \\
& \Rightarrow t^{2} s^{2}-s^{2}\|y, z\|^{2}+t^{2}\|x, z\|^{2}-\|x, z\|^{2}\|y, z\|^{2} \geq t^{2} s^{2}-t^{2}\|x, z\|^{2}+ \\
& s^{2}\|y, z\|^{2}- \\
& \|x, z\|^{2}\|y, z\|^{2} \\
& \Rightarrow t^{2}\|x, z\|^{2}-s^{2}\|y, z\|^{2} \geq 0
\end{aligned}
$$

Selanjutnya dari hubungan $\mathrm{s}+\mathrm{t} \geq\|x, z\|+\|y, z\| \geq\|x+y, z\|$, diperoleh

$V(x+y, z ; s+t)=\frac{(s+t)^{2}-\|x+y, z\|^{2}}{(s+t)^{2}+\|x+y, z\|^{2}} \geq \frac{(s+t)^{2}-(\|x, z\|+\|y, z\|)^{2}}{(s+t)^{2}+(\|x, z\|+\|y, z\|)^{2}}$

Selanjutnya

$$
\begin{aligned}
& \frac{(s+t)^{2}-(\|x, z\|+\|y, z\|)^{2}}{(s+t)^{2}+(\|x, z\|+\|y, z\|)^{2}}-\frac{s^{2}-\|x, z\|^{2}}{s^{2}+\|x, z\|^{2}} \\
& =\frac{\|x, z\|^{2}(s+t)^{2}-s^{2}(\|x, z\|+\|y, z\|)^{2}-s^{2}(\|x, z\|+\|y, z\|)^{2}+\|x, z\|^{2}(s+t)^{2}}{\left\{(s+t)^{2}+(\|x, z\|+\|y, z\|)^{2}\right\} .\left(s^{2}+\|x, z\|^{2}\right)}
\end{aligned}
$$

Sebut $A=\left\{(s+t)^{2}+(\|x, z\|+\|y, z\|)^{2}\right\} \cdot\left(s^{2}+\|x, z\|^{2}\right)$, maka

$$
=\frac{2}{A}\|\| x, z\left\|^{2}(s+t)^{2}-s^{2}(\|x, z\|+\|y, z\|)^{2}\right\|
$$$$
=\frac{2}{A}\left[\|x, z\|^{2} t^{2}+2 s t\|x, z\|^{2}-s^{2}\|y, z\|^{2}-2 s^{2}\|x, z\| \cdot\|y, z\|\right]
$$$$
=\frac{2}{A}\left[\|x, z\|^{2} t^{2}-s^{2}\|y, z\|^{2}+2 s\|x, z\|(t\|x, z\|-s\|y, z\|)\right] \geq 0 \text {, }
$$

(dari

(i))

Jadi 
$V(x+y, z ; s+t) \geq V(x, z ; t)$ jika $V(x, z ; t) \leq V(y, z ; t)$

Dengan cara yang sama akan diperoleh

$V(x+y, z ; s+t) \geq V(y, z ; t)$ jika $V(y, z ; t) \leq V(x, z ; t)$

Dengan demikian

$V(x+y, z ; s+t) \geq \min \{V(\mathrm{x}, z ; t), V(y, z ; t)\}$.

(FN-3.6). Ambil $t_{1}<\mathrm{t}_{2}$ akan ditunjukkan $V\left(x, y ; t_{1}\right) \leq V\left(x, y ; t_{2}\right)$, untuk setiap $x, y \in \mathrm{X}$.

Kasus 1. Misalkan $t_{2}>t_{1} \geq\|x, y\|$, maka perdefinisi diperolah $V\left(x, y ; t_{1}\right)$ $=V\left(x, y ; t_{2}\right)=0$.

Kasus 2. Misalkan $t_{1}<t_{2}<\|x, y\|$, maka berlaku :

$$
\begin{array}{r}
\frac{t_{2}^{2}-\|x, y\|^{2}}{t_{2}^{2}+\|x, y\|^{2}}-\frac{-\|x, y\|^{2}}{t_{1}^{2}+\|x, y\|^{2}}=\frac{t_{2}^{2}\|x, y\|^{2}-t_{1}^{2}\|x, y\|^{2}}{\left(t_{1}^{2}+\|x, y\|^{2}\right) \cdot\left(t_{2}^{2}+\|x, y\|^{2}\right)} \\
=\frac{\|x, y\|^{2}\left(t_{2}+t_{1}\right) \cdot\left(t_{2}-t_{1}\right)}{\left(t_{1}^{2}+\|x, y\|^{2}\right) \cdot\left(t_{2}^{2}+\|x, y\|^{2}\right)} \geq 0
\end{array}
$$

Jadi berlaku $V\left(x, y ; t_{2}\right)-V\left(x, y ; t_{1}\right) \geq 0,(x, y ;):.(0, \infty) \rightarrow[0,1]$ fungsi takturun untuk $t \in \mathrm{R}$.

(FN.3.7). Jelas $\lim _{t \rightarrow \infty} V(x, y ; t)=\lim _{t \rightarrow \infty} \frac{t^{2}-\|x, y\|^{2}}{t^{2}+\|x, y\|^{2}}=1$,

Maka $(X, V, *)$ merupakan ruang linear bernorm-2 fuzzy.

Juga mengacu pada definisi-definisi yang ada pada ruang fuzzy bernorma, didefinisikan berbagai definisi pada ruang fuzzy bernorm-2 sebagai berikut

Definisi 3.2. Misalkan $(X, V, *)$ ruang fuzzy bernorm-2, untuk $t>0$ dan unsur tak nol $z \in \mathrm{X}$, Himpunan bagian $\mathrm{A} \subseteq \mathrm{X}$ dikatakan t-terbatas jika terdapat $r \in(0,1)$ sehingga $\mathrm{V}(x, z ; t)>1-r$ untuk semua $x \in \mathrm{A}$.

Definisi 3.3. Untuk $t>0$ dan unsur tak nol $z \in X$. Barisan $\left\{x_{n}\right\}$ pada ruang fuzzy bernorma-2 $(X, V, *)$ dikatakan $t$-konvergen ke $x \in \mathrm{X}$, jika untuk setiap $0<\varepsilon<1$ terdapat $n_{0} \in \mathrm{N}$ sehingga untuk semua $n>n_{0}$ berlaku $V\left(x_{n}-x, z ; t\right)>1-\varepsilon$, dinotasikan dengan $x_{n} \stackrel{t}{\rightarrow} x$. 
Definisi 3.4. Untuk $t>0$ dan unsur tak nol $z \in X$. Barisan $\left\{x_{n}\right\}$ pada ruang fuzzy bernorma- $n(X, V, *)$ dikatakan $t$-konvergen ke $x \in \mathrm{X}$, jika untuk setiap $0<\varepsilon<1$ terdapat $n_{0} \in \mathrm{N}$ sehingga untuk semua $n>n_{0}$ berlaku $V\left(x_{n}-x_{k}, z ; t\right)>1-\varepsilon$,

Definisi 3.5. Misalkan $(X, V, *)$ ruang fuzzy bernorma-2, untuk $t>0$ dan unsur tak nol $z \in \mathrm{X}$, himpunan bagian $\mathrm{A} \subseteq \mathrm{X}$ dikatakan :

(i). $t$-tutup jika untuk setiap barisan $\left\{x_{n}\right\}$ di A konvergen ke suatu titik $x \in \mathrm{A}$

(ii). $t$-kompak setiap barisan $\left\{x_{n}\right\}$ di A mempunyai barisan bagian $\left\{x_{n_{k}}\right\}$ yang $t$ konvergen ke $x_{0} \in \mathrm{A}$.

Mengacu pada pendekatan ruang fuzzy bernorma dari (Kider, 2011a dan 2011b), maka bila didefinisikan untuk titik fuzzy $x_{\alpha}+y_{\beta}=(x+y)_{\lambda}$ dengan $\lambda=$ maks $\{\alpha, \beta\}$, maka dapat didefinisikan ruang fuzzy bernorma-2 sebagai berikut :

Definisi 3.6. Misalkan $\mathrm{X}$ ruang vector atas lapangan $\mathrm{K}$, dan misalkan $\|.,\|_{f}: \mathrm{X} \rightarrow$ $[0, \infty)$, fungsi yang mengaitkan setiap titik $x_{\alpha}, y_{\beta}$ di $X, \alpha, \beta \in(0,1]$ bilangan real tak negatip $\| .$, . $\|_{f}$ sehingga

(FN1). $\left\|x_{\alpha}, y_{\beta}\right\|_{f}=0$ jika dan hanya jika $x$ dan y bergantung linear.

(FN2). $\left\|x_{\alpha}, y_{\beta}\right\|_{f}=\left\|y_{\beta}, x_{\alpha}\right\|_{f}$ untuk semua $x_{\alpha}, y_{\beta}$ di X

(FN3). $\left\|\lambda x_{\alpha}, y_{\beta}\right\|_{f}=|\lambda|\left\|x_{\alpha}, y_{\beta}\right\|$, untuk semua $x_{\alpha}, \mathrm{y}_{\beta}$ di $\mathrm{X}$ dan semua $\lambda \in \mathrm{K}$.

(FN4). $\left\|x_{\alpha}+y_{\beta}, z_{\gamma}\right\|_{f} \leq\left\|x_{\alpha}, z_{\gamma}\right\|_{f}+\left\|y, z_{\gamma}\right\|_{f}$ untuk semua $x_{\alpha}, y_{\beta}$ dan $z_{\gamma}$ di X

(FN5). Jika $\left\|x_{\alpha}, y_{\beta}\right\|_{f}<r$, dengan $r>0$ maka terdapat $0<\alpha, \beta<\gamma, \rho \leq 1$ sehingga $\left\|x_{r}, y_{\rho}\right\|_{f}<r$.

Maka $\|., .\|_{f}$ disebut fuzzy norm-2 dan $\left(X .\|., .\|_{f}\right)$ disebut ruang fuzzy bernorma-2.

Berdasarkan Definisi 3.6 di atas, maka didapat hubungan antara rnorm biasa dengan fuzzy norm yaitu sebagai berikut. 
Proposisi 3.7. Misalkan $(X .\|.\|$,$) ruang bernorma biasa, definisikan \left\|x_{\alpha}, y_{\beta}\right\|_{f}=$ $\frac{1}{\delta}\|x, y\|$ dengan $\delta=$ maks $\{\alpha, \beta\}$,untuk semua $x_{\alpha}, y_{\beta}, z_{\gamma} \in X$ dan $\alpha, \beta, \gamma \in(0,1]$, maka $\left(X .\|.,\|_{f}\right)$ merupakan ruang fuzzy bernorma-2.

Bukti : Misalkan $x_{\alpha}, y_{\beta} \in \mathrm{X}$ dengan $\alpha, \beta \in(0,1]$ dan $\lambda \in \mathrm{K}$. maka :

(FN1). $\left\|x_{\alpha}, y_{\beta}\right\|_{f}=0 \Leftrightarrow \frac{1}{\delta}\|x, y\|=0 \Leftrightarrow\|x, y\|=0 \Leftrightarrow x$ dan $y$ bergantung linear.

(FN2). $\left\|x_{\alpha}, y_{\beta}\right\|_{f}=\frac{1}{\delta}\|x, y\|=\frac{1}{\delta}\|y, x\|=\left\|y_{\beta}, x_{\alpha}\right\|_{f}$

(FN3). $\left\|\lambda x_{\alpha}, y_{\beta}\right\|_{f}=\frac{1}{\delta}\|\lambda x, y\|=\frac{|\lambda|}{\delta}\|x, y\|=|\lambda| .\left\|x_{\alpha}, y_{\beta}\right\|_{f}$

(FN4). $\left\|x_{\alpha}+y_{\beta}, z_{\gamma}\right\|_{f} \leq\left\|(x+y)_{\tau}, z_{\gamma}\right\|_{f}, \quad$ dengan $\tau=\operatorname{maks}\{\alpha, \beta\}$

$$
\begin{array}{r}
=\frac{1}{\rho}\|x+y, z\| \leq \frac{1}{\rho}\|x, z\|+\frac{1}{\rho}\|y, z\|, \quad \text { dengan } \rho=\operatorname{maks}\{\tau, \gamma\} \\
\leq \frac{1}{\mu}\|x, z\|+\frac{1}{\vartheta}\|y, z\|, \quad \text { dengan } \mu=\operatorname{maks}\{\alpha, \gamma\} \text { dan } \varphi
\end{array}
$$

$=\operatorname{maks}\{\beta, \gamma\}$

$$
=\left\|x_{\alpha}, z_{\gamma}\right\|_{f}+\left\|y_{\beta}, z_{\gamma}\right\|_{f}
$$

(FN5). Jika $\left\|x_{\alpha}, y_{\beta}\right\|_{f}<r$, dengan $r>0$, karena $\delta=\operatorname{maks}\{\alpha, \beta\}$ dan untuk $\gamma, \rho \in$ $(0,1]$ dengan $\tau=$ maks $\{\gamma, \rho\}$ sehingga $\delta \leq \tau$, maka berlaku :

$$
\frac{\|x, y\|}{\tau} \leq \frac{\|x, y\|}{\delta}<r \text { yang bermakna }\left\|x_{\gamma} y_{\rho}\right\|_{f}<r .
$$

Sebaliknya berdasarkan Definisi 3.6 bila $\left\|x_{\alpha}, y_{\beta}\right\|_{f}$ adalah suatu Ruang fuzzy bernorma-2, definisikan $\|x, y\|=\left\|x_{1}, y_{\beta}\right\|_{f}$ atau $\|x, y\|=\left\|x_{\alpha}, y_{1}\right\|_{f}$, merupakan ruang bernorma-2. Jadi berdasarkan definisi 3.6 dan proposisi 3.7 diperoleh bila kita punya ruang bernorma-2, maka senantiasa bisa kita bentuk ruang fuzzy bernorma-2 dan begitu juga sebaliknya, dengan kata lain ruang bernorma-2 adalah ekivalen dengan ruang fuzzy bernorma-2. Sehingga semua sifat yang berlaku pada ruang bernorma juga akan berlaku pada ruang fuzzy bernorma.

Misalkan $\left(X .\|.\|_{f}\right)$ ruang fuzzy bernorma, definsikan 
$\left\|x_{\alpha}, y_{\beta}\right\|_{f}=\left\{\begin{array}{c}0, \quad j i k a\left\|x_{\alpha}-y_{\beta}\right\|_{f}=0 \\ \left\|x_{\alpha}\right\|_{f} \cdot\left\|y_{\beta}\right\|_{f}, \text { lainnya }\end{array}\right.$

$\operatorname{Maka}\left(X .\|.,\|_{f}\right)$ merupakan ruang fuzzy bernorma-2.

(FN.1). Jelas dari definisi, $\left\|x_{\alpha}, y_{\beta}\right\|_{f}=0$, jika dan hanya $x_{\alpha}, y_{\beta}$ bergantung linear.

(FN.2). jika $x_{\alpha}, y_{\beta}$ bergantung linear, jelas $\left\|x_{\alpha}, y_{\beta}\right\|_{f}=\left\|y_{\beta}, x_{\propto}\right\|_{f}$, selanjutnya misalkan tidak bergantung linear. Maka

$$
\left\|x_{\propto}, y_{\beta}\right\|_{f}=\left\|x_{\propto}\right\|_{f} \cdot\left\|y_{\beta}\right\|_{f}=\left\|y_{\beta}\right\|_{f} \cdot\left\|x_{\propto}\right\|_{f}=\left\|y_{\beta}, x_{\propto}\right\|_{f}
$$

(FN.3). $\left\|\lambda x_{\alpha}, y_{\beta}\right\|_{f}=\left\|\lambda x_{\alpha}\right\|_{f} \cdot\left\|y_{\beta}\right\|_{f}=|\lambda| \cdot\left\|x_{\alpha}\right\|_{f} \cdot\left\|y_{\beta}\right\|_{f}=|\lambda|\left\|x_{\alpha}, y_{\beta}\right\|_{f}$

(FN.4). $\left\|x_{\alpha}+y_{\beta}, z_{\gamma}\right\|_{f}=\left\|x_{\alpha}+y_{\beta}\right\|_{f} \cdot\left\|z_{\gamma}\right\|_{f} \leq\left(\left\|x_{\alpha}\right\|_{f}+\left\|y_{\beta}\right\|_{f}\right) \cdot\left\|z_{\gamma}\right\|_{f}$

$$
=\left\|x_{\alpha}\right\|_{f} \cdot\left\|z_{\gamma}\right\|_{f}+\left\|y_{\beta}\right\|_{f} \cdot\left\|z_{\gamma}\right\|_{f}=\left\|x_{\alpha}, z_{\gamma}\right\|_{f}+\left\|y_{\beta}, z_{\gamma}\right\|_{f}
$$

(FN.5). Jika $\left\|x_{\alpha}, y_{\beta}\right\|_{f}<r$, dengan $r>0$, maka

$\left\|x_{\alpha}\right\|_{f}<r$, jadi terdapat $0<\alpha<\gamma<1$ sehingga $\left\|x_{\gamma}\right\|_{f}<r$, dan

$\left\|y_{\beta}\right\|_{f}<r$, jadi terdapat $0<\beta<\delta<1$ sehingga $\left\|y_{\beta}\right\|_{f}<r$. Jadi

$\left\|x_{\gamma} y_{\delta}\right\|_{f}=\left\|x_{\gamma}\right\|_{f} \cdot\left\|y_{\beta}\right\|_{f}<r^{2}<r$

Jadi $\left\|x_{\alpha}, y_{\beta}\right\|_{f}$ merupakan norm-2 fuzzy pada $\mathrm{X}$ dan $\left(X .\|., .\|_{f}\right)$ merupakan ruang fuzzy bernorma-2.

\section{RUANG FUZZY BERNORMA- $N$.}

Berikut ini diberikan perumuman ruang fuzzy bernorma-n berdasarkan definisi 2.5 dan 3.1.

Definisi 4.1. Himpunan bagian fuzzy $\widetilde{A}$ pada $X^{n} \times R$ dikatakan norma- $n$ fuzzy pada $\mathrm{X}$ jika memenuhi :

(FN-n-1), $V\left(x_{1}, x_{2}, \ldots, x_{n} ; t\right)=0$, untuk semua $\mathrm{t} \in \mathrm{R}, \mathrm{t}<0$. 
(FN-n.2). $V\left(x_{1}, x_{2}, \ldots, x_{n} ; t\right)=1$, jika dan hanya jika $x_{1}, x_{2}, \ldots, x_{n}$ bergantung linear, dengan $\mathrm{t} \in \mathrm{R}, t>0$.

(FN-n.3). $V\left(x_{1}, x_{2}, \cdots, x_{n} ; t\right)$ invariant untuk semua permutasi dari $x_{1}, x_{2}, \ldots, x_{n}$

(FN-n.4). $V\left(x_{1}, x_{2}, \cdots, \alpha x_{n} ; t\right)=V\left(x_{1}, x_{2}, \cdots, x_{n} ; \frac{t}{\alpha}\right)$ untuk setiap $\alpha \neq 0$.

(FN-n.5). untuk semua $s, t \in \mathrm{R}$.

$$
\begin{aligned}
& V\left(x_{1}, x_{2}, \cdots, x_{n-1}, y+z ; s+t\right) \geq \\
& \min \left\{V\left(x_{1}, x_{2}, \cdots, x_{n-1}, y ; s\right), V\left(x_{1}, x_{2}, \cdots, x_{n-1}, z ; t\right)\right\}
\end{aligned}
$$

(FN-n.6). $V\left(x_{1}, x_{2}, \cdots, x_{n} ; t\right)$ fungsi tak turun untuk $t \in \mathrm{R}$.

(FN-n.7). $\lim _{t \rightarrow \infty} V\left(x_{1}, x_{2}, \cdots, x_{n} ; t\right)=1$

Maka $V$ dikatakan dikatakan norma- $n$ fuzzy pada $\mathrm{X}$ dan pasangan $(X, V, *)$ dikatakan ruang fuzzy bernorma- $n$.

Mengacu pada contoh ruang fuzzy bernorma, maka Bila $(X,\|\bullet, \bullet, \cdots, \bullet\|)$ ruang bernormaa-n, definisikan $\mathrm{a} * \mathrm{~b}=\mathrm{ab}$ atau $\mathrm{a} * \mathrm{~b}=\min \{\mathrm{a}, \mathrm{b}\}$ dan

$$
\begin{gathered}
V\left(x_{1}, x_{2}, \cdots, x_{n} ; t\right)= \\
\left\{\begin{array}{cl}
\frac{k t^{n}}{k t^{n}+m\left\|x_{1}, x_{2}, \cdots, x_{n}\right\|} & \text { bila } k, m, n, t>0, \text { dan } x_{1}, x_{2}, \cdots, x_{n} \in X \\
0, & \text { bila } t \leq 0, \text { dan } x, y \in R
\end{array}\right.
\end{gathered}
$$

Maka $(X, V, *)$ merupakan ruag fuzzy bernorma- $n$. Khusus jika $k=n=m=1$, maka diperoleh bentuk

$$
V\left(x_{1}, x_{2}, \cdots, x_{n} ; t\right)=\left\{\begin{array}{cl}
\frac{t}{1+\left\|x_{1}, x_{2}, \cdots, x_{n}\right\|} & \text { bila } t>0, \text { dan } x_{1}, x_{2}, \cdots, x_{n} \in X \\
0, & \text { bila } t \leq 0, \text { dan } x, y \in R
\end{array}\right.
$$

Definisi 4.2. Misalkan $(X, V, *)$ ruang fuzzy bernorma- $n$, untuk $t>0$ dan unsur tak nol $\mathrm{z} \in \mathrm{X}$, Himpunan bagian $\mathrm{A} \subseteq \mathrm{X}$ dikatakan t-terbatas jika terdapat $r \in(0,1)$ sehingga $\mathrm{V}\left(x_{1}, x_{2}, \cdots, x_{n-1}, \mathrm{z} ; \mathrm{t}\right)>1-r$ untuk semua $x \in \mathrm{A}$. 
Definisi 4.3. Untuk $t>0$ dan unsur tak nol $z \in X$. Barisan $\left\{x_{n}\right\}$ pada ruang fuzzy bernorma- $n(X, V, *)$ dikatakan t-konvergen ke $x \in \mathrm{X}$, jika untuk setiap $0<\varepsilon<1$ terdapat $n_{0} \in \mathrm{N}$ sehingga untuk semua $\mathrm{n}>\mathrm{n}_{0}$ berlaku $\mathrm{V}\left(x_{1}, x_{2}, \cdots, x_{n-1}, \mathrm{x}_{\mathrm{n}}-\mathrm{x}, ; \mathrm{t}\right)>1-$ $\varepsilon$, dinotasikan dengan $x_{n} \stackrel{t}{\rightarrow} x$.

Definisi 4.4. Untuk $\mathrm{t}>0$ dan unsur tak nol $z \in \mathrm{X}$. Barisan $\left\{x_{n}\right\}$ pada ruang fuzzy bernorma- $n(X, V, *)$ dikatakan t-konvergen ke $x \in \mathrm{X}$, jika untuk setiap $0<\varepsilon<1$ terdapat $n_{0} \in \mathrm{N}$ sehingga untuk semua $n>n_{0}$ berlaku $V\left(x_{1}, x_{2}, \cdots, x_{n-1}, x_{m}-x_{k}, t\right)>1$ $-\varepsilon$,

Kembali ke ruang fuzzy bernorma- $n$ yang merupakan pengembangan dari definisi 3.5, sedikit ramai notasi untuk unsur-unsur di $\tilde{A}$, bila $x_{1}, x_{2}, \ldots, x_{n} \in \mathrm{X}$, maka fuzzy point di $\tilde{A}$ mestinya dinotasikan dengan $x_{1_{\alpha_{1}}}, x_{2_{\alpha_{2}}, \ldots, x_{n_{\alpha_{n}}}}$ dengan $0<\alpha_{\mathrm{i}} \leq 1, \mathrm{i}=$ $1,2, \ldots, n$. Dalam tulisan ini penulisan unsur titik fuzzy disederhanakan yaitu unsur $x_{1_{\ltimes_{1}}}, x_{2_{\alpha_{2}}, \cdots, x_{n_{\alpha_{n}}}}$ menjadi $x_{\alpha_{1}}, x_{\alpha_{2}}, \cdots, x_{\alpha_{n}}$ yang bermakna unsur $x_{\mathrm{i}} \in \mathrm{X}$ berkorespondensi dengan $x_{i_{\alpha_{i}}} \in \tilde{A}$.

Mengacu pada Definisi 3.6, maka didefinisikan ruang linear bernorma- $n$ fuzzy sebagai berikut :

Definisi 4.5. Misalkan $\mathrm{X}$ ruang vektor atas lapangan $\mathrm{K}$, dan misalkan $\|., \cdots, .\|_{f}: \mathrm{X}$ $\rightarrow[0, \infty)$, fungsi yang mengaitkan setiap titik $x_{\alpha_{1}}, x_{\alpha_{2}}, \cdots, x_{\alpha_{n}}$ di $\widetilde{A}, \alpha_{i} \in(0,1]$ bilangan real tak negatif $\|., \cdots, .\|_{f}$ sehingga

(FN1). $\left\|x_{\alpha_{1}}, x_{\alpha_{2}}, \cdots, x_{\alpha_{n}}\right\|_{f}=0$ jika dan hanya jika $x_{1}, x_{2}, \cdots, x_{n}$ bergantung linear (FN2). $\left\|x_{\alpha_{1}}, x_{\alpha_{2}}, \cdots, x_{\alpha_{n}}\right\|_{f}$ invariat terhadap permutasi

(FN3). $\quad\left\|\lambda x_{\alpha_{1}}, x_{\alpha_{2}}, \cdots, x_{\alpha_{n}}\right\|_{f}=|\lambda|\left\|x_{\alpha_{1}}, x_{\alpha_{2}}, \cdots, x_{\alpha_{n}}\right\|_{f} \quad, \quad$ untuk $\quad$ semua $x_{\alpha_{1}}, x_{\alpha_{2}}, \cdots, x_{\alpha_{n}}$ di $\mathrm{X}$ dan semua $\lambda \in \mathrm{K}$. 
(FN4). $\left\|x_{\alpha}+y_{\beta}, x_{\alpha_{2}}, \cdots, x_{\alpha_{n}}\right\|_{f} \leq\left\|x_{\alpha}, x_{\alpha_{2}}, \cdots, x_{\alpha_{n}}\right\|_{f}+\left\|y_{\beta}, x_{\alpha_{1}}, x_{\alpha_{2}}, \cdots, x_{\alpha_{n}}\right\|_{f}$ untuk semua $x_{\alpha}, \mathrm{y}_{\beta}$ dan $x_{\alpha_{2}}, \cdots, x_{\propto_{n}}$ di X

(FN5). Jika $\left\|x_{\alpha_{1}}, x_{\alpha_{2}}, \cdots, x_{\propto_{n}}\right\|_{f}<r$, dengan $r>0$ maka terdapat $0<\alpha_{\mathrm{i}}<\beta_{\mathrm{j}} \leq 1, i, j$ $=1,2, \ldots, n$ sehingga $\left\|x_{\beta_{1}}, x_{\beta_{2}}, \cdots, x_{\beta_{n}}\right\|_{f}<r$.

Maka $\|., \cdots, .\|_{f}$ disebut fuzzy norm-2 dan $\left(\mathrm{X}, \widetilde{A},\|., \cdots, .\|_{f}\right)$ disebut ruang fuzzy bernorma- $n$.

Ekivalen dengan proposisi 3.7, maka kalau bila $\left\|x_{1}, x_{2}, \cdots, x_{n}\right\|$ ruang linear bernorma- $n$, maka kalau didefinisikan $\left\|x_{\alpha_{1}}, x_{\alpha_{2}}, \cdots, x_{\alpha_{n}}\right\|_{f}=\frac{1}{\delta}\left\|x_{1}, x_{2}, \cdots, x_{n}\right\|$ dengan $\delta=$ maks $\left\{\alpha_{1}, \alpha_{2}, \ldots, \alpha_{n}\right\}$ maka dengan cara yang sama dengan pembuktian teorema 3.6 akan diperoleh $\left\|x_{\alpha_{1}}, x_{\alpha_{2}}, \cdots, x_{\alpha_{n}}\right\|_{f}$ ruang linear bernorma- $n$ fuzzy. Begitu juga sebaliknya jika $\left\|x_{\alpha_{1}}, x_{\alpha_{2}}, \cdots, x_{\alpha_{n}}\right\|_{f}$ ruang linear bernorma- $n$ fuzzy, maka kalau didefinisikan $\left\|x_{1}, x_{2}, \cdots, x_{n}\right\|=\left\|x_{i}, x_{\alpha_{2}}, \cdots, x_{\alpha_{n}}\right\|_{f}$ dengan $\mathrm{i}=1$ dan $\alpha_{\mathrm{j}}$ $\neq$ i. atau dalam notasi lain $\left\|x(i), x_{\alpha_{2}}, \cdots, x_{\alpha_{n}}\right\|_{f}$ dengan $\alpha_{j} \neq 1$, maka $\left\|x_{1}, x_{2}, \cdots, x_{n}\right\|=\left\|x(1), x_{\alpha_{2}}, \cdots, x_{\alpha_{n}}\right\|_{f}$ merupakan ruang linear bernorma-n. Jadi untuk pendefinisian di atas, ruang linear bernorma- $n$ adalah ekivalen dengan linear fuzzy bernorma- $n$. Sehingga konsep yang berlaku pada ruang linear bernorma- $n$.juga akan berlaku pada ruang linear fuzzy bernorma- $n$.

Mengacu pada (Gunawan dan Mashadi, 2001a dan 2001b), bahwa kalau $\left\|x_{1}, x_{2}, \cdots, x_{n}\right\|$ norma- $n$ pada $\mathrm{X}$, maka senantiasa dapat dibentuk $\left\|x_{1}, x_{2}, \cdots, x_{n-1}\right\|$ yang merupakan norma-(n-1) pada $\mathrm{X}$ dan begitu juga sebaliknya. Jadi kalau kita punya ruang fuzzy bernorma- $n$, maka juga senantiasa ak/an dapat dibentuk norma-( $n$ 1) pada $X$ dan begitu juga sebaliknya. 
Teorma 4.6. Misalkan $\left\|x_{\alpha_{1}}, x_{\alpha_{2}}, \cdots, x_{\propto_{n}}\right\|_{f}$ norma-n fuzzy pada X, kemudian bentuk

$$
\left\|x_{\alpha_{1}}, x_{\alpha_{2}}, \cdots, x_{\alpha_{n-1}}\right\|_{f}=\operatorname{maks}\left\|x_{\alpha_{1}}, x_{\alpha_{2}}, \cdots, x_{\alpha_{n}-1}, a_{\beta_{i}}\right\|_{f}
$$

dengan $\left\{a_{1}, a_{2}, \cdots, a_{n}\right\}$ himpunan yang bebas linear di X. yang berkorespondensi dengan unsur $\left\{a_{\beta_{1}}, a_{\beta_{2}}, \cdots, a_{\beta_{n}}\right\} \quad$ di $\widetilde{A}, \quad$ Maka $\left\|x_{\alpha_{1}}, x_{\alpha_{2}}, \cdots, x_{\alpha_{n-1}}\right\|_{f}$ norm-(n-1) fuzzy pada $X$.

Bukti : Bukti (FN.1) s/d (FN.4) ekivalen dengan bukti teorema 2.1 pada [7], maka disini akan dibuktikan (FN.5) saja.

$$
\text { Misalkan } \quad\left\|x_{\alpha_{1}}, x_{\alpha_{2}}, \cdots, x_{\propto_{n-1}}\right\|_{f}=\operatorname{maks}\left\|x_{\alpha_{1}}, x_{\alpha_{2}}, \cdots, x_{\alpha_{n}-1}, a_{\beta_{j}}\right\|_{f}<r,
$$

dengan $r>0$, karena $\left\|x_{\alpha_{1}}, x_{\alpha_{2}}, \cdots, x_{\alpha_{n}}\right\|_{f}$ norma- $n$ fuzzy, maka terdapat $\gamma_{\mathrm{i}}$ dan $\delta_{\mathrm{i}}$ dengan $\alpha_{\mathrm{i}}<\gamma_{\mathrm{i}}$ dan $\beta_{\mathrm{i}}<\delta_{\mathrm{j}} \quad \mathrm{i}=1,2, \ldots, n$-1, sehingga $\left\|x_{\gamma_{1}}, x_{\gamma_{2}}, \cdots, x_{\gamma_{n-1}}\right\|_{f}=$ maks $\left\|x_{\gamma_{1}}, x_{\gamma_{2}}, \cdots, x_{\gamma_{n}-1}, a_{\delta_{j}}\right\|_{f}<r$. Jadi $\left\|x_{\alpha_{1}}, x_{\alpha_{2}}, \cdots, x_{\alpha_{n-1}}\right\|_{f}$ norma-(n-1) fuzzy pada $X$.

\section{DAFTAR PUSTAKA}

Alotaibi, A. M, (2010), On Statisticall Convergent Double Sequences in Intutionistic Fuzzy 2-Normed Spaces, 4(46), 2249 - 2262.

Bag. T dan Samanta. S.K, (2005), Fuzzy Bounded Linear Operator, Fuzzy Set and Sustems, 151, $513-547$.

Elegan, S.K, Zayen, E.M.W dan Noval, T, A. (2010), Some Remarks on Series in Fuzzy n-Normed Spaces, Int Math Forum, 5(3), 117 - 124.

Eshaghi. M.G, Abbaszadeh. S dan Rassias, Th. M, (2009), On the Mazur-Ulam Theorem In Fuzzy n-Normed Strictly Convex Spaces, Acxiv math, FA, 1 - 7. 
Golet.I, (2009), On Generalized fuzzy normed spaces, Int Math Forum, 4(25), 1237 1242.

Gunawan, H dan Mashadi, (2001a), On Finite Dimensional 2-Normed Spaces, Soocow J of Math, 7(3), 321-329

Gunawan, H dan Mashadi, (2001b), On n-Normed Space, IJMMS, 27(10), 631 - 639

Iqbal H. Jebril, and Hemen Dutta, (2010), Generalization of n-Normed Space, General Mathematics Notes, I(1), 8 - 19.

Karakus S, Demirki K and Duman O, (2008), Statistical Convergence on Intituinistic Fuzzy Linear Space, Chaos Solitons and Fractals, 35, 763769.

Kider J.R, (2011), On Ruang fuzzy bernormas, Eng \& Tech Journal, 29(9), 1790 1795.

Kider J.R, (2011), Completion of Fuzzy Normed Space, Eng \& Tech Journal, 29(10), $2004-2012$.

Mashadi. (2010), A New Method for Dual Fully Fuzzy Linear System by Use LUFactorizations of the Coefficient Matrix, JMS, 17(3), $101-106$.

Moghaddam. M.A dan Sistani. T, (2011), On t-Best Coapproximation in Fuzzy 2Normed Spaces, 5(9), $2241-2248$.

Mursaleen. M dan Danish Lohani. Q.M,(2008), Intuitionistic Fuzzy 2-Normed Space and Some Related Concepts, Chaos, Solitons and Fracta,

Saadi. R dan Vaezpour. S.M., (2005), Some Result on Banach Spaces, J.Appl Math \& Computing, 17(no 1-2), $475-484$.

Samanta. T.K and Jebril. I.H, (2009), Finite Dimentional Intuitunionistic Fuzzy Normed Linear Space, Int J Open Problems Comp math, 2(4), 574 - 591. 
Samanta. T.K dan Mohinta. S, (2011), A Note on Generalizwd Intuitionistic Fuzzy $\psi$ Normed Linear Space, Global J of Science Frontier Research, 11(1), 1 - 13.

Sharma. S, (2002), On Fuzzy Metric Space, Southeast Asian Bull of Math, 26, 133 145 .

Somasundaram. R.M dan Beaula. T, (2009), Some Aspects of 2-Fuzzy 2-Normed Linear Space, Bull of the Malaysian Math Scie Soc, 2, 32(2), 211 - 221.

Surender Teddy. B, (2011), Intuitionistic Fuzzy 2-Norm, Int Journal of Math Analysis, 5(14), $651-659$.

Vaezpour.S.M dan Karimi. F, (2008), T-Best Approximation in Ruang fuzzy bernorma, 5(2), $93-99$.

Vilayabalaji. S, Natesan. T dan Jun. Y. B, (2007), Intuitionistic Fuzzy $n$-Normed Linear Spaces, Bull Korean Math Soc, 44(2), 291 - 308..

Xiao. J. Z dan Zhu, X.H. (2003), Ruang fuzzy bernorma of Operator and its Completeness, Fuzzy Set and Systems, 133, 389 - 399. 